\title{
Comparison of myocardial reperfusion between intracoronary versus intravenous cangrelor administration in patients undergoing primary percutaneous coronary intervention
}

\author{
Iacopo Muraca ${ }^{1}$,, Matteo Pennesi ${ }^{1}$, Alessio Mattesini ${ }^{2}$, Angela Migliorini ${ }^{1}$, \\ Nazario Carrabba ${ }^{1}$, Giacomo Virgili ${ }^{3}$, Filippo Bruscoli ${ }^{3}$, Pierluigi Demola ${ }^{2}$, \\ Riccardo Colombi ${ }^{3}$, Giulia Pontecorboli ${ }^{1}$, Niccolò Marchionni ${ }^{3}$, \\ Carlo Di Mario ${ }^{2}$, Renato Valenti ${ }^{1}$
}

${ }^{1}$ Interventional Cardiology Unit, Cardiovascular Department, Careggi University Hospital, Florence, Italy ${ }^{2}$ Structural Intervention Cardiology Unit, Cardiovascular Department, Careggi University Hospital, Florence, Italy

${ }^{3}$ Department of Clinical and Experimental Medicine, Careggi University Hospital, Florence, Italy

\begin{abstract}
Background: Myocardial reperfusion is the main target of treatment in patients with ST-segment elevation myocardial infarction (STEMI). The intracoronary administration of cangrelor bolus could favor a higher local drug concentration, favoring an earlier thrombotic resolution and a reduced distal micro-embolization.

Methods: Seventy-one patients undergoing primary percutaneous coronary intervention (PCI) for STEMI: 37 treated with intracoronary and 34 with intravenous bolus administration of cangrelor. The primary endpoint was ST-segment elevation resolution (STR) $\geq 50 \%$ after 30 min from the end of the $P C I$. Other explorative reperfusion indices investigated were: $S T R \geq 50 \%$ at 24 hours, $S T R \geq 70 \%$ at 30 min, Thrombolysis In Myocardial Infarction frame count and the QT dispersion. Moreover, acute and subacute stent thrombosis, bleeding events and 30-day mortality have been evaluated.

Results: More frequent STR $\geq 50 \%$ was observed in the intravenous cangrelor bolus group as compared to the intracoronary administration at $30 \mathrm{~min}(71.9 \% \mathrm{vs.} 45.5 \% ; p=0.033)$, the difference was maintained 24 hours after PCI (87.1\% vs. 63.6\%; $p=0.030)$. STR $\geq 70 \%$ at 30 min was statistically more frequent in the intravenous bolus administration cohort $(66.7 \%$ vs. $28.6 \% p=0.02)$. At multivariable analysis, intravenous cangrelor administration was significantly related to $S T R \geq 50 \%$ (odds ratio: 3.586; 95\% confidence interval: 1.134-11.335; $p=0.030$ ). The incidence of Bleeding Academic Research Consortium 3-5 bleedings was $15.5 \%$ and mortality was $4.2 \%$ without any significant difference between the two groups.

Conclusions: In conclusion the results of the study do not show any advantages in the administration of intracoronary bolus of cangrelor in patients affected by STEMI and treated with primary PCI. (Cardiol J)
\end{abstract}

Key words: primary percutaneous coronary intervention, ST-segment elevation myocardial infarction, cangrelor, intracoronary bolus administration, ST-segment elevation resolution, myocardial reperfusion

Address for correspondence: Renato Valenti, MD, Interventional Cardiology, Careggi University Hospital, Largo Brambilla 3, 50141 Florence, Italy, tel: +39 055794 9094, e-mail: renato.valenti2@tin.it

Received: 24.07.2021 Accepted: 7.08.2021 Early publication date: 23.09.2021

This article is available in open access under Creative Common Attribution-Non-Commercial-No Derivatives 4.0 International (CC BY-NC-ND 4.0) license, allowing to download articles and share them with others as long as they credit the authors and the publisher, but without permission to change them in any way or use them commercially. 


\section{Introduction}

Myocardial reperfusion is the main target of the treatment strategy in patients with ST-segment elevation myocardial infarction (STEMI). The most powerful oral P2Y12-receptor inhibitors, prasugrel and ticagrelor, showed a several hour time-frame delay in their activity onset, especially in patients presenting with STEMI [1]. Thus, in this setting it is crucial to have an intravenous drug available with a faster and intense platelet inhibition. The use of antithrombotic glycoprotein IIb/IIIa receptor inhibitors (GPI) is associated with a high bleeding risk, and currently their use should be considered for bailout situations (evidence of no-reflow or thrombotic complications) or angiographic evidence of massive intracoronary thrombosis during percutaneous coronary intervention (PCI).

Cangrelor is an intravenous $\mathrm{P} 2 \mathrm{Y} 12$ receptor antagonist and it reaches its therapeutic concentration within minutes. It is indicated in patients undergoing PCI, to reduce ischemic adverse events, in patients who have not received oral P2Y12 inhibitors before PCI or where oral P2Y12 inhibition is not feasible or desirable. Cangrelor binds the P2Y12 receptor directly, so it does not need hepatic activation [2]; half-life is short, about $3-5 \mathrm{~min}$, and it is rapidly inactivated by hematic dephosphorilation [3-5]. It reversibly inhibits the P2Y12 receptor after an initial bolus dose of $30 \mu \mathrm{g} / \mathrm{kg}$ followed by continuous infusion of $4 \mu \mathrm{g} / \mathrm{kg} /$ $/ \mathrm{min}$. The infusion is indicated for at least 2 hours or until the end of the procedure, whichever is longer. Platelet aggregation inhibition is achieved within a few minutes, and its restoration is achieved within 60-90 min after stopping the administration [2]. Thus, cangrelor is ideal in STEMI patients treated with primary PCI, because there is little time for pre-treatment with oral P2Y12 inhibitors and increased thrombotic risk. The safety and efficacy of cangrelor was investigated in 3 pivotal randomized clinical trials of the CHAMPION trial program (Cangrelor versus Standard Therapy to Achieve Optimal Management of Platelet Inhibition) and in the pooled analysis [6-8]. Patients with STEMI were only about $12 \%$ of the study population in CHAMPION, but with a consistent $19 \%$ relative reduction of the composite primary end point. More recently, the pharmacodynamic evidence effects of cangrelor in combination with ticagrelor for patients treated with primary PCI was investigated in the CANTIC study (Platelet Inhibition with Cangrelor and Crushed Ticagrelor in STEMI Patients Undergoing Primary Percutaneous Coronary Intervention) [9].
Intracoronary administration of abciximab or other GPI during PCI constitutes a safe approach for drug administration and was associated with a significant improvement in myocardial perfusion despite no clear clinical benefit $[10,11]$. Similarly, the intracoronary administration of cangrelor could favor a higher local drug concentration, leading to a deeper platelet inhibition in the clinical setting of primary PCI. This could be associated with an earlier thrombotic resolution and a reduced distal micro-embolization translating in myocardial reperfusion improvement. However, no data exists about the intracoronary administration of cangrelor in the management setting of primary PCIs.

The aim of this prospective study was to compare myocardial reperfusion assessed by ST-segment elevation resolution (STR) provided by intracoronary bolus or intravenous administration of cangrelor.

\section{Methods}

Cangrelor became available in our institution from the beginning of 2019. In a prospective and nonrandomized fashion; thus, the study is exploratory and should be considered mainly as hypothesis generating, all consecutive patients enrolled were admitted for STEMI within 12 hours from symptoms onset treated with primary PCI, without any restriction based on age or clinical status at presentation. Patients with a history of oral P2Y12 inhibitor therapy or treated with clopidogrel, prasugrel or ticagrelor before the procedure or with anticoagulant were excluded. Other exclusion criteria were: administration of intravenous thrombolysis or use of an extracorporeal life support device; major surgery $<6$ weeks; stroke $<30$ days or any history of hemorrhagic stroke; participation in another study and patients presenting pacemaker rhythm or with left bundle block.

According to institutional protocol, after the confirmed diagnosis of STEMI, patients received intracoronary or intravenous cangrelor bolus after diagnostic coronary angiography, at operator's discretion.

Selective coronary angiography was performed in multiple projections before mechanical reperfusion. The preferred access was radial. Immediately after diagnostic angiography, antithrombotic and anticoagulant treatment was administered and primary PCI intervention with drug eluting stenting of the infarct-related vessel was performed using standard material. Mechanical thrombectomy was performed at the operators' 
discretion in case of massive intracoronary thrombosis and in case of thrombotic complications. Successful primary PCI was defined as Thrombolysis In Myocardial Infarction (TIMI) grade 3 coronary flow in the treated vessel with a residual stenosis $<20 \%$. Acetylsalicylic acid was administered to all patients before primary PCI (150 to $300 \mathrm{mg}$ orally or $150 \mathrm{mg}$ intravenously). During the procedure, intravenous unfractioned heparin was administered accordingly to achieve an activated clotting time of 250-300 s. Cangrelor bolus dose was administered as recommended according to patient weight ( $30 \mu \mathrm{g} / \mathrm{kg}$ bolus followed by $4 \mu \mathrm{g} / \mathrm{kg} / \mathrm{min}$ continuous infusion for $2 \mathrm{~h}$ ) after the diagnostic angiography by central/peripheral vein or intracoronary through the guiding catheter after the cannulation of culprit infarct-related artery. After the procedure, cangrelor infusion was continued by central/peripheral vein in all patients to reach the two recommended hours of treatment. At the end of the procedure, all patients received a loading dose of ticagrelor (180 mg) followed by $90 \mathrm{mg}$ twice a day.

Primary end point of the study was a surrogate of myocardial reperfusion: the early STR $\geq 50 \%$ at $30 \mathrm{~min}$ after primary PCI. Other explorative surrogates of myocardial reperfusion included: a) the STR $\geq 70 \%$ at 30 min after PCI; b) the STR $\geq$ $50 \%$ at 24 hours after PCI; c) QT dispersion (QTd); d) post-procedural corrected TIMI frame count.

Electrocardiogram (ECG) was recorded 30 -min and 24 hours after the procedure, using the same electrocardiograph. The ST-segment changes were evaluated in the single lead with the most prominent ST-segment elevation before mechanical intervention. The ST-segment elevation was measured to the nearest $0.5 \mathrm{~mm}$ at $60 \mathrm{~ms}$ after the J point with the aid of a hand-held caliper. The STR was defined as a reduction in ST-segment elevation $\geq 50 \%$ or $\geq 70 \%$ at $30 \mathrm{~min}$ and at 24 hours after primary PCI. To obtain the QTd measure, the QT interval length of every lead where it was easily readable (using the diagonal method) was measured, then every QT interval length was corrected (QTc) using the Bazett formula; finally, the difference between longest QTc and shortest one was calculated. TIMI frame count was measured considering the time in frames taken by the contrast to pass from the proximal to the distal part of the culprit vessel; the frame speed was 15 frames/s. We considered first frame to be when the contrast touched both the vessel walls and matted at least $70 \%$ of the lumen. The distal markers position depended on the coronary: in the right coronary artery it was the first collateral branch of the posterior-lateral vessel; in the left anterior descending coronary artery (LAD) was the "whale tale"; in the circumflex coronary artery was at the more distal part of the branches for the obtuse margin. The obtained frames have been doubled to correct the acquisition time from 15 frames/s to $30 \mathrm{frames} / \mathrm{s}$, according to available literature. For the $\mathrm{LAD}$, the total number of frames was divided by 1.7 (correction factor).

All electrocardiographics and angiographic markers of reperfusion analyses were performed by physicians blinded to the cangrelor bolus administration route.

Adverse clinical outcomes within 30 days were collected including all-cause mortality, cardiovascular mortality, myocardial reinfarction, stroke, acute and sub-acute stent thrombosis according to the Academic Research Consortium [12, 13], urgent target vessel revascularization, unplanned revascularization, bleeding events according to Bleeding Academic Research Consortium (BARC) classification [14], and if clinically relevant (the need for blood transfusion or a reduction of hemoglobin more than $3 \mathrm{~g} / \mathrm{dL}$ ).

The study complied with the Declaration of Helsinki and Good Clinical Practice, and was approved by the institutional ethics committee, and patients or legal representatives signed written informed consent.

Patients were divided in two groups according to the cangrelor bolus administration (intravenous or intracoronary). Discrete data are expressed as frequencies, and continuous data as mean \pm standard deviation or median and interquartile range, as appropriate. The $\chi^{2}$ test was used to compare categorical variables, and the unpaired two-tailed Student t-test or Mann-Whitney rank-sum test was used to test differences between continuous variables. The univariable and multivariable analyses to evaluate the independent contribution of the variables to the primary and other explorative surrogate of myocardial reperfusion end points were performed by the binary logistic regression model. The variables that reached the highest significance at the univariable analysis were considered in the final multivariable model in order to avoid overfitting. The following variables were considered: mean age, female, LAD as the culprit lesion, chronic kidney disease, diabetes, intraprocedural thrombectomy and intracoronary or intravenous cangrelor bolus administration. In order to minimize bias due to the nonrandomized nature of the study and the possibility of overfitting, a propensity score analysis was performed using 
Table 1. Baseline characteristics.

\begin{tabular}{lcccc}
\hline & Overall $(\mathbf{n}=\mathbf{7 1})$ & Intracoronary $(\mathbf{n}=37)$ & Intravenous $(\mathbf{n}=34)$ & $\mathbf{P}$ \\
\hline Age [years] & $65.6 \pm 13.3$ & $67.1 \pm 13$ & $64.12 \pm 13.6$ & 0.563 \\
Female & $15(21.1 \%)$ & $9(24.3 \%)$ & $6(17.6 \%)$ & 0.491 \\
Familiar history of CAD & $21(29.6 \%)$ & $11(32.4 \%)$ & $10(27 \%)$ & 0.623 \\
Hypertension & $40(56.3 \%)$ & $24(64.9 \%)$ & $16(47.1 \%)$ & 0.131 \\
Dyslipidemia & $30(42.3 \%)$ & $14(37.8 \%)$ & $16(47.1 \%)$ & 0.432 \\
Diabetes & $12(16.9 \%)$ & $6(16.2 \%)$ & $6(17.6 \%)$ & 0.862 \\
Active smokers & $39(54.9 \%)$ & $23(62.2 \%)$ & $16(47.1 \%)$ & 0.201 \\
PAD & $7(9.9 \%)$ & $4(10.8 \%)$ & $3(8.8 \%)$ & 0.779 \\
Previous PCl & $11(15.5 \%)$ & $5(13.5 \%)$ & $6(17.6 \%)$ & 0.631 \\
CKD & $10(14.1 \%)$ & $6(16.2 \%)$ & $4(11.76 \%)$ & 0.590 \\
Baseline ST-segment elevation: & & & & 0.273 \\
Mean \pm SD [mm] & $2.79 \pm 1.83$ & $2.80 \pm 1.61$ & $2.78 \pm 2.07$ & 0.720 \\
Median [IQR] [mm] & $2.5[1.5-3.5]$ & $2.75[1.62-3.5]$ & $2.25[1.5-3.88]$ &
\end{tabular}

CAD — coronary artery disease; CKD — chronic kidney disease; IQR — interquartile range; PAD — peripheral artery disease; PCI — percutaneous coronary intervention; SD — standard deviation

a logistic regression model from which the probability for the intracoronary or intravenous bolus cangrelor administration was calculated for each patient; variables introduced into propensity score model were: age (years), female, diabetes, previous coronary revascularization, pre-procedural ST-segment elevation, and LAD coronary artery as the culprit vessel. Model discrimination was assessed with the C-statistic and goodness-of-fit with Hosmerand-Lemeshow test. Thereafter, a multivariable analysis was performed using the propensity score as a continuous covariate. Binary logistic multivariate analysis was used to test interaction between thrombectomy and intravenous or intracoronary cangrelor bolus administration. To compare the measures of the QTd and the TIMI frame count between the groups, the values above the median and above the $75^{\circ}$ percentile were analyzed. All tests were two-tailed. A p value $<0.05$ was considered significant. Analyses were performed with SPSS statistical package, Version 21 (IBM Corp., Armonk, NY, USA).

\section{Results}

Out of 220 STEMI patients from March 2019 to August 2020, 77 were treated with cangrelor and 71 were available for the analysis: 34 patients in the intravenous bolus administration group, and 37 patients in the intracoronary group (excluded patients: 4 had ventricular pace-maker complex, 2 had previous left bundle branch block).
Among the 71 patients who underwent cangrelor administration during primary $\mathrm{PCI}$, baseline characteristics were well balanced between the two groups (Table 1). Mean age was $65 \pm 13$, there were $21 \%$ of female patients. Diabetic patients accounted for the $17 \%$ of the study population and $15.5 \%$ of the patients had previous coronary revascularization. Angiographic and procedural characteristics are depicted in Table 2. At baseline ECG, there was no difference between the median values of ST-segment elevation in the intracoronary and intravenous groups $(2.80 \pm 1.61 \mathrm{~mm}$ vs. $2.78 \pm 2.07 \mathrm{~mm}$; $\mathrm{p}=0.273$ ). Radial access was used in $67.6 \%$ of patients; LAD was the culprit vessel in $40(56.3 \%)$ cases. Intra-procedural rheolytic thrombectomy use differed between the two groups: $50 \%$ of cases in intravenous bolus group vs. $13.4 \%$ in intracoronary bolus group $(\mathrm{p}=0.002)$. Primary PCI was successful in all patients of the study cohort (post procedural TIMI 3 with a residual stenosis $<20 \%$ ). All patients received second generation drug eluting stents (Table 2). In all patients (100\%) oral P2Y12 inhibitor was ticagrelor.

The early STR $\geq 50 \%$ primary end point was significantly higher in intravenous than intracoronary group ( $71.9 \%$ vs. $45.5 \%$; $p=0.033$ ) (Fig. 1 ). At univariable analysis, LAD as culprit vessel showed an inverse correlation with early STR (odds ratio [OR] $0.283 ; 95 \%$ confidence interval [CI] 0.097-0.828; $\mathrm{p}=0.021)$ as well as chronic kidney disease (OR 0.159; 95\% CI 0.030-0.838; $\mathrm{p}=0.030$ ). At multivariable analysis, intravenous cangrelor 
Table 2. Angiographic and procedural characteristics.

\begin{tabular}{|c|c|c|c|c|}
\hline & Overall $(n=71)$ & Intracoronary $(n=37)$ & Intravenous $(n=34)$ & $\mathbf{P}$ \\
\hline Culprit vessel: & & & & 0.941 \\
\hline LAD & $40(56.3 \%)$ & $20(54.1 \%)$ & $20(58.8 \%)$ & \\
\hline $\mathrm{CX}$ & $5(7.0 \%)$ & $2(5.4 \%)$ & $3(8.8 \%)$ & \\
\hline $\mathrm{RCA}$ & $26(36.6 \%)$ & $14(37.8 \%)$ & $12(35.3 \%)$ & \\
\hline TIMI 0-1 & $55(78 \%)$ & $27(74 \%)$ & $28(82 \%)$ & 0.344 \\
\hline Multivessel CAD & $44(62 \%)$ & $24(64 \%)$ & $20(59 \%)$ & 0.274 \\
\hline Type B2 and C lesion & $68(97 \%)$ & $35(96 \%)$ & $33(98 \%)$ & 0.946 \\
\hline \multicolumn{5}{|l|}{ Thrombectomy: } \\
\hline Rheolytic & $21(29.6 \%)$ & $5(13.4 \%)$ & $16(50 \%)$ & 0.002 \\
\hline Manual & $3(4.2 \%)$ & $1(2.9 \%)$ & $2(5.5 \%)$ & 0.506 \\
\hline Stent recipients & $71(100 \%)$ & $37(100 \%)$ & $34(100 \%)$ & \\
\hline \multicolumn{5}{|l|}{ Type of stents: } \\
\hline Second generation EES ( $n$ ) & 103 & 45 & 58 & \\
\hline Number of stents per patients & $1.42 \pm 0.66$ & $1.24 \pm 0.59$ & $1.61 \pm 0.69$ & \\
\hline Ischemia time [h] & $3.6 \pm 2.0$ & $3.5 \pm 2.0$ & $3.6 \pm 2.1$ & 0.838 \\
\hline \multicolumn{5}{|l|}{ Vascular access: } \\
\hline Radial & $48(67.6 \%)$ & $30(81.1 \%)$ & $18(52.9 \%)$ & 0.11 \\
\hline Femoral & $23(32.4 \%)$ & $7(18.9 \%)$ & $16(47.1 \%)$ & 0.11 \\
\hline \multicolumn{4}{|l|}{ P2Y12 post } & 1.00 \\
\hline Ticagrelor & $71(100 \%)$ & $37(100 \%)$ & $34(100 \%)$ & 1.00 \\
\hline
\end{tabular}

CAD - coronary artery disease; CX - circumflex, IQR — interquartile range; EES - everolimus eluting stent; LAD — left anterior descending; RCA - right coronary artery

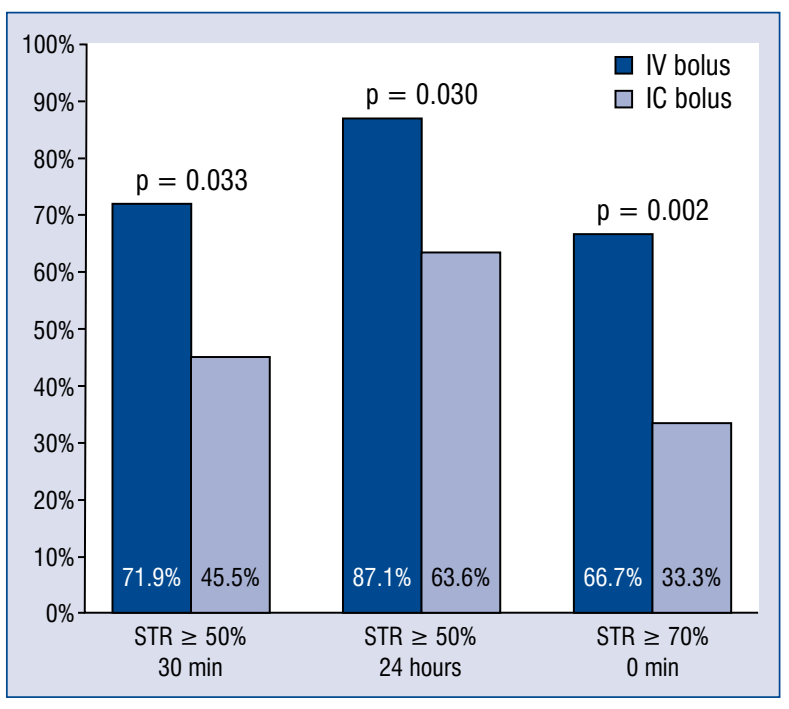

Figure 1. ST-segment elevation resolution (STR) as surrogate of myocardial reperfusion; IC — intracoronary; IV - intravenous.

bolus administration was significantly related to STR $\geq 50 \%$ (OR 3.586; 95\% CI 1.134-11.335; $\mathrm{p}=0.030)$ and remained significantly associated with the primary end point also after propensity score adjustment $(\mathrm{OR} 3.23 ; \mathrm{p}=0.032 ; \mathrm{C}$-statistic $=$ $=0.67 ; \mathrm{p}=0.977$ for Hosmer-Lemeshow test) (Fig. 2).

STR $\geq 50 \%$ was evaluated also at 24 hours after the procedure as another explorative surrogate index of myocardial reperfusion, and intravenous bolus administration of cangrelor resulted in the only variable associated with STR in the multivariable analysis (OR 4.250; 95\% CI 1.128-16.016; $\mathrm{p}=0.033$ ). Similar results were obtained with $30 \mathrm{~min}$ STR $\geq 70 \%$ (OR $5.591 ; 95 \%$ CI 1.859-16.819; $\mathrm{p}=0.002$ ). No interaction was found between thrombectomy and intracoronary or intravenous cangrelor bolus administration $(p=0.762)$. No differences were found between the two treatment strategies regarding post procedural TIMI frame count ( $30.08 \pm 18.1$ vs. $26.8 \pm 13.4 ; \mathrm{p}=0.258$ ), even analyzing the sub-group with LAD as culprit $\operatorname{vessel}(25.1 \pm 11.4$ vs. $25.5 \pm 14.9$; $\mathrm{p}=0.710)$. Also, no differences were found considering the median and the $75^{\circ}$ percentile dichotomous cut-off values for the analyses ( $p=0.390$ and $p=0.525$, respectively).

QTd analysis showed similar results between the two study cohorts early at $30 \mathrm{~min}$ after the 


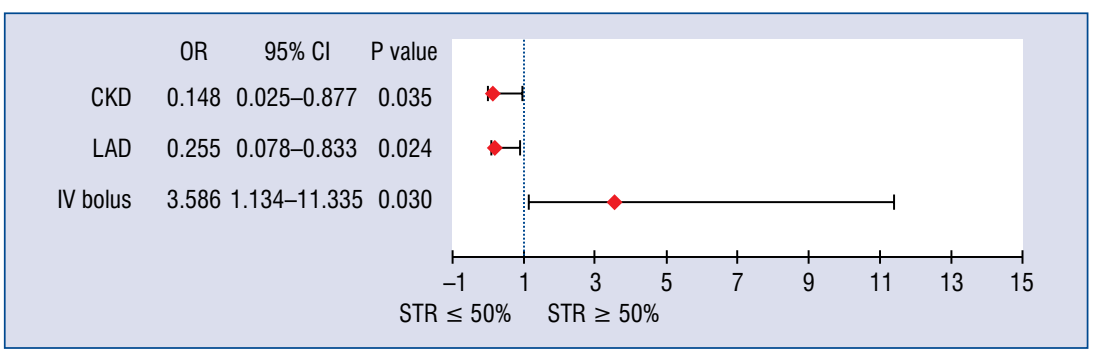

Figure 2. Adjusted analysis of predictors associated with early ST-segment elevation resolution (STR) $\geq 50 \% ; \mathrm{Cl}-$ confidence interval; CKD — chronic kidney disease; IV — intravenous; LAD — left anterior descending; OR — odds ratio.

primary PCI procedure $(57.7 \pm 26.5 \mathrm{~ms}$ vs. $57.1 \pm$ $\pm 27.7 \mathrm{~ms} ; \mathrm{p}=0.880)$ and at 24 hours $(53.1 \pm 24.4$ vs. $58.1 \pm 21.9 ; \mathrm{p}=0.591$ ).

No ischemic events or stent thrombosis occurred at 30 days. Two (5.4\%) patients in the intracoronary group and $1(2.9 \%)$ patient in the intravenous group experienced cardiac death, due to refractory heart failure, before hospital discharge $(\mathrm{p}=0.606)$. All bleedings and BARC $\geq 3$ bleeding events were not related to intracoronary or intravenous cangrelor bolus administration (Table 2); 11 BARC $\geq 3$ bleeding events occurred in the study population (15\%), and out of these, 7 (63\%) were in the femoral procedures. No other clinical events occurred.

\section{Discussion}

The main findings of the study can be summarized as follows: 1 ) intracoronary administration of cangrelor was not associated with an improved myocardial reperfusion; conversely, intravenous bolus administration was associated with a significantly improved reperfusion assessed by an early and late STR; 2) no acute or sub-acute stent thrombosis occurred, and 3) no differences in terms of clinically relevant/significant bleedings (BARC $3-5$ ) were found between groups.

Intravenous cangrelor bolus administration was associated with a greater early STR $\geq 50 \%$ and other surrogate indices of myocardial reperfusion. Intravenous bolus administration resulted independently associated with STR, even after the multivariable adjustment for the most important clinical variables. The association was significant for $30 \mathrm{~min}$ STR $\geq 50 \%$ and $30 \mathrm{~min}$ STR $\geq 70 \%$, and for 24 hours STR $\geq 50 \%$, which is known to be related to myocardial reperfusion and salvage [15-20].

The mechanisms involved and the explanation of this phenomenon are not completely clear.
It could be hypothesized that the result could be favored by different timing of drug administration: the intracoronary administration by guiding catheter after the cannulation of the coronary "culprit" vessel could lead to a delay of action of the drug as compared to an earlier conventional intravenous bolus administration, which is performed immediately after the diagnostic coronary angiography. Thus, it can be supposed that an earlier administration could overcome a higher local drug concentration. In particular, the mechanical instrumentation by primary PCI of the "culprit" vessel, immediately after the intracoronary bolus, would occur without an adequate platelet inhibition by cangrelor. This could favor distal embolization and micro-embolization phenomena; as known, micro-embolization plays a crucial role in the intracoronary flow pathophysiology, leading to microvascular thrombosis, vasoconstriction and myocardial inflammation [21]. These pathophysiologic effects are associated with clinical outcome and prognosis. According to available literature, the absence of STR after the procedure is associated with microcirculation dysfunction [22], leading to greater myocardial damage and increased cardiovascular mortality and reinfarction at 1 and 3 years [20,23].

Therefore, the small sample size derived data herein, support how different routes of cangrelor administration could affect myocardial reperfusion in primary PCI, and consequently the clinical outcome of patients.

As previously reported, in the present population study, non-LAD coronary artery "culprit" vessel was associated with a higher rate of STR $[19,24]$. In the study by Schröder [19], comparing different methods of STR, the anterior acute myocardial infarction was associated with less reduction in STR; furthermore, the group of patients considered to be at low risk for complete resolution of ST-segment elevation, included more 
than two-thirds of the total number of patients with inferior acute myocardial infarction [19]. The randomized JETSTENT multicenter trial, compared rheolytic thrombectomy before direct stenting to direct stenting alone in STEMI patients undergoing primary PCI. The study that assessed myocardial reperfusion by early STR, showed a significant inverse correlation of anterior acute myocardial infarction with early resolution of the ST-segment elevation by multivariable analysis [24].

Thrombectomy was more frequently used in the intravenous bolus cohort of the study. However, it was not related to STR in univariable and multivariable analyses. In accordance to the current guidelines [25], thrombectomy was employed in patients with a large or massive thrombus-containing lesion and/or in bailout situations. Furthermore, no interaction was found between thrombectomy and intracoronary or intravenous bolus of cangrelor administration.

Renal insufficiency (defined as estimated glomerular filtration rate $<60 \mathrm{~mL} / \mathrm{min}$ ) was associated with a worse myocardial reperfusion, as shown by univariable and multivariable analysis. Renal impairment does not affect pharmacocynetic and pharmacodynamic of cangrelor. Instead, it is possible that the known negative effect of the renal insufficiency on the endothelial function and its pro-thrombotic effects, other than the favorable calcium deposition in the coronary circle, could be associated with an impairment of myocardial reperfusion. In available literature, data show that renal insufficiency is a predictor of worse postprocedural coronary flow (measured by the TIMI flow grade) after reperfusion [26, 27]; these data could correlate with a low rate of STR.

Finally, the small sample size of the pilot study prevents inferences about the exploratory clinical outcomes. Nevertheless, no concerning results emerged about the safety with the intracoronary bolus administration of cangrelor.

\section{Limitations of the study}

The present study must be evaluated in light of several limitations. Firstly, data derived from a single-center prospective registry. The study was mechanistic in nature and was not powered for clinical end points. Despite the use of multivariable analysis, it remains unknown if residual confounders may have affected the results in the present analyses. Another limitation was the number of patients that made type II errors possible. It must be acknowledged that this study did not show a cause-and-effect relationship, but only an association. Thus, the results of this study should be considered only as hypothesis generating.

\section{Conclusions}

In conclusion, the results of our study do not show any advantages from the administration of intracoronary bolus of cangrelor in patients affected by STEMI and treated with primary PCI.

\section{Acknowledgments}

The authors are indebted to the CathLab, Operating Room and CCU staffs for their precious help. We are also very grateful to Fabio Torrini and Paola Baldini (A.R. Card Onlus Foundation; Florence; Italy) for their secretarial assistance.

This work has been supported by a donation offered to Fondazione A.R. Card Onlus in memory of Marco Sarti by his beloved wife and son, relatives and friends.

\section{Conflict of interest: None declared}

\section{References}

1. Parodi G, Valenti R, Bellandi B, et al. Comparison of prasugrel and ticagrelor loading doses in ST-segment elevation myocardial infarction patients: RAPID (Rapid Activity of Platelet Inhibitor Drugs) primary PCI study. J Am Coll Cardiol. 2013; 61(15): 1601-1606, doi: 10.1016/j.jacc.2013.01.024, indexed in Pubmed: 23500251.

2. Akers WS, Oh JJ, Oestreich JH, et al. Pharmacokinetics and pharmacodynamics of a bolus and infusion of cangrelor: a direct, parenteral P2Y12 receptor antagonist. J Clin Pharmacol. 2010; 50(1): 27-35, doi: 10.1177/0091270009344986, indexed in Pubmed: 19779037.

3. Franchi F, Rollini F, Muñiz-Lozano A, et al. Cangrelor: a review on pharmacology and clinical trial development. Expert Rev Cardiovasc Ther. 2013; 11(10): 1279-1291, doi: 10.1586/14779072.2013.837701, indexed in Pubmed: 24138516.

4. Waite LH, Phan YL, Spinler SA. Cangrelor: a novel intravenous antiplatelet agent with a questionable future. Pharmacotherapy. 2014; 34(10): 1061-1076, doi: 10.1002/phar.1471, indexed in Pubmed: 25123696.

5. Ferri N, Corsini A, Bellosta S. Pharmacology of the new P2Y12 receptor inhibitors: insights on pharmacokinetic and pharmacodynamic properties. Drugs. 2013; 73(15): 1681-1709, doi: 10.1007/s40265-013-0126-z, indexed in Pubmed: 24114622.

6. Abtan J, Steg PG, Stone GW, et al. Efficacy and safety of cangrelor in preventing periprocedural complications in patients with stable angina and acute coronary syndromes undergoing percutaneous coronary intervention: the CHAMPION PHOENIX trial. JACC Cardiovasc Interv. 2016; 9(18): 1905-1913, doi: 10.1016/j.jcin.2016.06.046, indexed in Pubmed: 27659566.

7. Cavender MA, Bhatt DL, Stone GW, et al. Consistent Reduction in Periprocedural Myocardial Infarction With Cangrelor as Assessed by Multiple Definitions: Findings From CHAMPION PHOENIX 


\section{Cardiology Journal}

(Cangrelor Versus Standard Therapy to Achieve Optimal Management of Platelet Inhibition). Circulation. 2016; 134(10): 723733, doi: 10.1161/CIRCULATIONAHA.115.020829, indexed in Pubmed: 27482008.

8. Groves EM, Bhatt DL, Steg PG, et al. Incidence, Predictors, and Outcomes of Acquired Thrombocytopenia After Percutaneous Coronary Intervention: A Pooled, Patient-Level Analysis of the CHAMPION Trials (Cangrelor Versus Standard Therapy to Achieve Optimal Management of Platelet Inhibition). Circ Cardiovasc Interv. 2018 Apr; 11(4): e005635, doi: 10.1161/CIRCINTERVENTIONS.117.005635, indexed in Pubmed: 29632238.

9. Franchi F, Rollini F, Rivas A, et al. Platelet inhibition with cangrelor and crushed ticagrelor in patients with ST-segment-elevation myocardial infarction undergoing primary percutaneous coronary intervention. Circulation. 2019; 139(14): 1661-1670, doi: 10.1161/CIRCULATIONAHA.118.038317, indexed in Pubmed: 30630341.

10. Stone GW, Maehara A, Witzenbichler B, et al. Intracoronary abciximab and aspiration thrombectomy in patients with large anterior myocardial infarction: the INFUSE-AMI randomized trial. JAMA. 2012; 307(17): 1817-1826, doi: 10.1001/jama.2012.421, indexed in Pubmed: 22447888.

11. De Luca G, Verdoia M, Suryapranata H. Benefits from intracoronary as compared to intravenous abciximab administration for STEMI patients undergoing primary angioplasty: a meta-analysis of 8 randomized trials. Atherosclerosis. 2012; 222(2): 426-433, doi: 10.1016/j.atherosclerosis.2012.02.041, indexed in Pubmed: 22483166.

12. Cutlip DE, Windecker S, Mehran R, et al. Clinical end points in coronary stent trials: a case for standardized definitions. Circulation. 2007; 115(17): 2344-2351, doi: 10.1161/CIRCULATIONAHA.106.685313, indexed in Pubmed: 17470709.

13. Cutlip DE, Nakazawa G, Krucoff MW, et al. Autopsy validation study of the academic research consortium stent thrombosis definition. JACC Cardiovasc Interv. 2011; 4(5): 554-559, doi: 10.1016/j.jcin.2011.01.011, indexed in Pubmed: 21596329.

14. Mehran R, Rao SV, Bhatt DL, et al. Standardized bleeding definitions for cardiovascular clinical trials: a consensus report from the Bleeding Academic Research Consortium. Circulation. 2011; 123(23): 2736-2747, doi: 10.1161/CIRCULATIONAHA.110.009449, indexed in Pubmed: 21670242.

15. Schröder R, Dissmann R, Brüggemann T, et al. Extent of early ST segment elevation resolution: a simple but strong predictor of outcome in patients with acute myocardial infarction. J Am Coll Cardiol. 1994; 24(2): 384-391, doi: 10.1016/0735-1097(94)90292-5, indexed in Pubmed: 8034872.

16. van't Hof AW, Liem A, de Boer MJ, et al. Clinical value of 12-lead electrocardiogram after successful reperfusion therapy for acute myocardial infarction. Lancet. 1997; 350(9078): 615-619, doi: 10.1016/s0140-6736(96)07120-6.
17. Santoro GM, Valenti R, Buonamici P, et al. Relation between ST-segment changes and myocardial perfusion evaluated by myocardial contrast echocardiography in patients with acute myocardial infarction treated with direct angioplasty. Am J Cardiol. 1998; 82(8): 932-937, doi: 10.1016/s0002-9149(98)00508-6, indexed in Pubmed: 9794347.

18. de Lemos JA, Braunwald E. ST segment resolution as a tool for assessing the efficacy of reperfusion therapy. J Am Coll Cardiol. 2001; 38(5): 1283-1294, doi: 10.1016/s0735-1097(01)01550-9.

19. Schröder R. Prognostic impact of early ST-segment resolution in acute ST-elevation myocardial infarction. Circulation. 2004; 110(21): e506-e510, doi: 10.1161/01.CIR.0000147778.05979.E6, indexed in Pubmed: 15557381.

20. Farkouh ME, Reiffel J, Dressler O, et al. Relationship between ST-segment recovery and clinical outcomes after primary percutaneous coronary intervention: the HORIZONS-AMI ECG substudy report. Circ Cardiovasc Interv. 2013; 6(3): 216-223, doi: 10.1161 /CIRCINTERVENTIONS.112.000142, indexed in Pubmed: 23652600.

21. Heusch G, Kleinbongard P, Böse D, et al. Coronary microembolization: from bedside to bench and back to bedside. Circulation. 2009; 120(18): 1822-1836, doi: 10.1161/CIRCULATIONAHA.109.888784, indexed in Pubmed: 19884481.

22. Feldman LJ, Coste P, Furber A, et al. Incomplete resolution of ST-segment elevation is a marker of transient microcirculatory dysfunction after stenting for acute myocardial infarction. Circulation. 2003; 107(21): 2684-2689, doi: 10.1161/01. CIR.0000070423.91346.45, indexed in Pubmed: 12742980.

23. van der Zwaan HB, Stoel MG, Roos-Hesselink JW, et al. Early versus late ST-segment resolution and clinical outcomes after percutaneous coronary intervention for acute myocardial infarction. Neth Heart J. 2010; 18(9): 416-422, doi: 10.1007/ BF03091808, indexed in Pubmed: 20862236.

24. Antoniucci D. JETSTENT trial results: impact on ST-segment elevation myocardial infarction interventions. J Invasive Cardiol. 2010; 22(10 Suppl B): 23B-25B, indexed in Pubmed: 20947933.

25. Neumann F-J, Sousa-Uva M, Ahlsson A, et al. 2018 ESC/EACTS Guidelines on myocardial revascularization. Eur Heart J. 2019 Jan 7; 40(2): 87-165, doi: 10.1093/eurheartj/ehy394, indexed in Pubmed: 30165437.

26. Polanska-Skrzypczyk M, Karcz M, Bekta P, et al. Prognostic value of renal function in STEMI patients treated with primary PCI: ANIN Registry. Br J Cardiol. 2013; 20: 65, doi: 10.5837/ bjc.2013.17.

27. Sabroe JE, Thayssen P, Antonsen L, et al. Impact of renal insufficiency on mortality in patients with ST-segment elevation myocardial infarction treated with primary percutaneous coronary intervention. BMC Cardiovasc Disord. 2014; 14: 15, doi: 10.1186/1471-2261-14-15, indexed in Pubmed: 24506974. 